\title{
Fear of snakes in wild- and laboratory-reared rhesus monkeys (Macaca mulatta)
}

\author{
SUSAN MINEKA, RICHARD KEIR, and VEDA PRICE \\ University of Wisconsin, Madison, Wisconsin 53706
}

\begin{abstract}
Experiment 1 compared the responses of 10 laboratory-reared and 10 wild-reared rhesus monkeys (Macaca mulatta) to a real snake and to a range of snake-like objects. Most wildreared monkeys showed considerable fear of the real, toy, and model snakes, whereas most lab-reared monkeys showed only very mild responses. Fear was indexed by unwillingness to approach food on the far side of the snake and by behavioral disturbance. Experiment 2 examined the effectiveness of seven flooding sessions in reducing snake fear in 8 wild-reared rhesus monkeys. Mean latency to reach for food, trials to criterion (four consecutive short latency responses), and total exposure time to criterion declined significantly across flooding sessions. Behavioral disturbance declined within sessions but not across sessions. Results of a final behavioral test revealed that substantial long-lasting changes had occurred in only 3 of the 8 monkeys. The resuits are discussed in the context of dissociation between different indices of fear.
\end{abstract}

It has long been known that many adult primate species exhibit a marked fear of snakes. Some investigators who have studied this fear have suggested, or assumed, that it was "innate" or "spontaneous" (e.g., Hebb, 1946; Masserman \& Pechtel, 1953; Morris \& Morris, 1965). These terms are only meant to imply that the fear does not appear to result from any specific experience, although obviously maturational and/or general life experience factors may play a role in its development. Other investigators, by contrast, have argued that some form of learning or social tradition is likely to be involved in the origins of this fear. Yerkes and Yerkes (1936), for example, argued that their data supplied "no evidence of specific avoidance (fear) response prior to or apart from individual experience with a given type of object [e.g., snake]" (p. 64). They reached their conclusions largely because adult chimpanzees exhibited greater fear in their reaction to snakes than did infant chimpanzees (see also Haselrud, 1938). Schiller (1952) reached a similar conclusion regarding fear of snakes when he wrote "there is no evidence in the ape of innate specific responses to specific perceptual configurations [e.g., snakes]" (p. 182). His argument was based on results that showed differences in the rate of formation of approach and avoidance reactions to snakes and to other, more arbitrary, neutral objects.

This research was supported in part by grants to the first author from the University of Wisconsin Graduate School and by Grant MH-27156 from the U.S. Public Health Service. The authors would like to thank Antonio Gino, Robert Hendersen, Carole Mohr, J. Bruce Overmier, and Jack Rachman for their help in various phases of this research. Requests for reprints should be sent to Susan Mineka, Department of Psychology, 1202 W. Johnson Street, Madison, Wisconsin 53706.
A somewhat more direct strategy for getting at the origins of snake fear than that used by Yerkes, Haselrud, or Schiller involves comparing the responses of lab-reared primates with those of wildreared primates. Although both Hebb (1946) and Yerkes and Yerkes (1936) had a mixture of wildand captive-reared chimpanzees in their studies, no systematic comparisons were made between the responses of the two groups. Furthermore, Yerkes and Yerkes noted that all their subjects had probably had experience with snakes even in their captive environment and that there was a good possibility that they had learned the fear. More recently, Joslin, Fletcher, and Emlen (1964) systematically compared the responses of wild-and lab-reared rhesus monkeys to snakes and snake-like objects; the lab-reared monkeys had been raised indoors and had not had any experience with snakes. Their results indicated that only the wild-reared monkeys consistently exhibited a strong fear of snakes (either a live 2- $\mathrm{ft}$ bullsnake or lifelike models). Although the lack of fear in the lab-reared monkeys could be an aberration from normal development or the result of habituation to snake-like objects (e.g., hoses) in their laboratory surroundings, Joslin et al. thought that an alternative interpretation was more likely. They suggested that, to have become afraid of snakes, wild-reared monkeys had had some necessary learning experience that lab-reared monkeys had not had, that is, that the fear is not "innate" or "spontaneous," but learned. The plausibility of this explanation was supported by Stephenson's (1967) experiment, which demonstrated that rhesus monkeys could learn by observation to fear relatively arbitrary objects. In a study similar to that of Joslin et al. (1964), Murray and King (1973) showed that there were systematic differences in wild- 
and lab-reared squirrel monkeys' fear of snakes. Wildreared, but not lab-reared, squirrel monkeys displayed significantly longer food-reach latencies when presented with a live snake or a snake-like object than when presented with a neutral object.

Although considerable attention has been devoted to documenting the existence of a pronounced fear reaction to snakes in many primate species and to the attempts discussed above to ascertain whether the fear is spontaneous or learned, very little attention has been directed to the question of how easy it is to modify this fear. Schiller (1952) observed that his chimpanzees' fear of snakes diminished quite rapidly when, in a counterconditioning procedure, a snake was paired with a banana. However, these results are difficult to evaluate for a number of reasons. First, Schiller did not specify how long each of the counterconditioning trials lasted (only "until the banana was taken," p. 181), and it is possible that a large amount of exposure was required before the fear reaction was "abolished." Second, the snake was presented in a glass box with a glass cover, on top of which the banana was placed. Thus, the chimpanzees may have learned simply to not fear snakes enclosed in glass boxes. Finally, Schiller provided no follow-up data to indicate if the effects were long-lasting or if there was substantial spontaneous recovery.

In the only other published attempt to modify snake fear, Murray and King (1973) exposed their squirrel monkeys to the snake and a snake-like object three times a day for 14 consecutive days. Their trials were of short duration $(60 \mathrm{sec})$ and terminated when the monkey reached for the food. The results indicated that the food-reach latencies and the total amount of time spent in visually scanning the objects declined steadily across days. Unfortunately, these results are somewhat difficult to interpret for several reasons. First, their monkeys were severely food deprived $(23 \mathrm{~h})$ at the time of testing and so had considerable incentive to reach for the food (which we cannot even be sure, from their description, was on the far side of the snake). Second, because their trials terminated as soon as the monkeys reached for the food, it is possible that their subjects were, in fact, learning an escape response. Third, their results on visual scanning (not a very dramatic fear behavior) are reported in terms of total time rather than as a percentage of the trial, and so are confounded by the varying lengths of their trials. Finally, because they did not run any follow-up tests, they provided, like Schiller, no data indicating the permanence of their effects.

The present experiments were designed to further explore, in rhesus monkeys, some of the issues discussed above. Experiment 1 provided a replication and slight extension of the Joslin et al. (1964) study.
In this experiment, reactions of wild- and lab-reared rhesus monkeys to a live boa constrictor and to other snake-like objects were compared. The procedure differed from that of Joslin et al. in that the live snake was presented unrestrained in an open box rather than in clamps. This was done in order to determine whether the confinement procedure used by Joslin et al. might have minimized the level of fear exhibited in their lab-reared monkeys. In addition, all objects were presented twice, rather than only once as in the Joslin et al. study, in order to help determine the extent to which the simple novelty of the stimulus objects played a role in the results they obtained. In Experiment 2, eight wildreared rhesus monkeys that had shown a fear of snakes were given seven flooding or extinction sessions over a period of 35 days in order to assess the degree to which fear of snakes could be reduced and how long-lasting any reductions might be.

\section{EXPERIMENT 1}

\section{Method}

\section{Subjects}

The subjects were 20 male and female rhesus monkeys (Macaca mulotta). Ten were born and raised in the Wisconsin Primate Laboratory, and they ranged in age from 8 to 21 years (mean = 13.5). The remaining 10 were born in the wild and imported from India 10 to 23 years ago. Their approximate ages ranged from 15 to 29 years $($ mean $=21.6)$. Three members of each group (wild- and lab-reared) were male, and the other seven in each group were female. Life history records were checked in an attempt to determine which, if any, of the animals might have had previous experiences that could have biased their performance in this study. The majority had clearly had WGTA experience, but, because of incomplete records, it was impossible to be sure whether all had had such experience. One lab-reared monkey had been a subject in the Joslin et al. (1964) study and so had had previous exposure to a snake and to snake-like objects (no treatment or conditioning was involved). The monkeys had all participated in a range of experiments over the years (e.g., learning. mother surrogate, sexual behavior, steroid metabolism, etc.) and had lived in a range of conditions (single cage, peer groups, nuclear family).

\footnotetext{
Apparatus

The following objects were used to determine each subject's reactions: (1) A young, 2- to $2 \frac{1}{2} 2$-ft-long $(60-75 \mathrm{~cm})$ live boa constrictor (Constrictor constrictor), approximately 1 in. $(2.6 \mathrm{~cm})$ in diameter; (2) a nonmoving, 20-in.-long $(56 \mathrm{~cm})$, sinuous, lifelike model of a snake, approximately $1 / 2$-in. $(1.3 \mathrm{~cm})$ in diameter and colored to imitate the appearance of a grass snake; (3) a 24 -in.-long $(61 \mathrm{~cm})$, sinuous, rubber toy snake, approximately 1 in. $(2.6 \mathrm{~cm})$ in diameter, that jiggled slightly when moved; (4) a 3-f:-long $(91.5 \mathrm{~cm})$, sinuous, black rubber electrical cord, approximately $3 / 8 \mathrm{in.}(1 \mathrm{~cm})$ in diameter; (5) a $33 / 4-f t-l o n g$ $(114 \mathrm{~cm})$, sinuous, yellow plastic-covered electrical cord; $(6)$ an unpainted square wood block; and (7) a red, triangular, wood block.

All tests were performed in the Wisconsin Primate Laboratory using a Wisconsin General Test Apparatus (WGTA). The WGTA consists of a cage for the subject, a movable cage blind, a movable gray tray used to present stimulus objects, and a one-
} 
way mirror behind which the experimenter sits (see Harlow, 1949. for a detailed description). The objects were all presented in one of tho uncovered $53.4 \times 21.3 \times 15.2 \mathrm{~cm}$ Plexiglas boxes, each with a small Plexiglas ledge at the top of the back side (side away from the monkey) where a food reward could be placed The Plexiglas box was placed on the movable gray tray.

\section{Procedure}

Within 3 days betore the test day, each subject was adapted to the apparatus and procedure. Although many or all of the subjects had had previous WGTA experience, they were all readapted in order to ensure that their performance would be comparable. The adaptation technique used was similar to that used in the general adaptation procedure for the WGTA. On the day before the adaptation day, the animal was fed only approx. imately one-third of its usual ration. The subject was not fed again until after it had completed the adaptation session the next day. A food reward (either a fruit loop or a marshmallow, depending on individual preference) was placed on the ledge of the Plexiglas box. An adaptation object (one of two different, colored, wood blocks) was placed in the box. The moving tray with the Plexiglas box on it was then advanced toward the subject. The subject was considered to be adapted when it reached for and touched the food reward within $5 \mathrm{sec}$ on 9 of 10 trials. Most subjects required several days of adaptation before reaching criterion.

On the day before the testing session, each subject was once more fed only approximately one-third of its usual ration. The subject was not fed again until after it was tested.

In the testing session, (1) the cage blind was lowered, (2) one of the test objects was placed in the Plexiglas box with the subject's preferred food reward (determined during adaptation) on the ledge, (3) the cage blind was raised, and (4) the tray was pushed toward the subject. An electric timer was started when the tray had been pushed completely forward. The timer was stopped when the subject touched the food reward. It should be noted that with this procedure the monkey was required to reach across the open box containing the test object in order to obtain the reward. The trial was terminated after $60 \mathrm{sec}$ if the subject had not yet reached for the food.

There were 22 trials, which included 2 presentations of each of the five snake-like objects and 12 presentations of the neutral objects. The first 2 trials were always with the neutral wood objects. The remaining five snake-like objects were presented in one of two predetermined orders that differed in whether the real or the toy snake was presented first: (1) toy snake, yellow cord, model snake, black cord, real snake, or (2) real snake, yellow cord, model snake, black cord, toy snake. Half of each group of subjects (wild- and lab-reared) received the first order; the remaining half received the second order. One of the two wood objects was always presented between each of the other objects, the two wood objects being used alternately. The stimuli were then repeated in the same order as first presented, starting with the presentation of the two wood objects and then proceeding with a wood object between each of the other test stimuli.
The latency of the food-reaching response and the emotional responses that occurred during that latency period were observed and recorded. The emotional responses were scored on the basis of behavioral scoring techniques used widely in the Wisconsin Primate Laboratory (e.g., Suomi, 1974). Pilot data had indicated that there were 10 emotional-disturbance behaviors likely to occur. These behaviors were: cage clutch (holding onto side or back of cage), cage shake (obvious moving or shaking of cage), eye aversion (rapidly looking away from stimulus), fear grimace (stretching the lips over the gums, thus exposing the teeth), spasm/tic (a vigorous shaking or jerking of the hands or upper body), threat (lips thrust forward, ears retracted and flattened against head), vocalization, fear withdraw (sudden retreat to the back of the cage), ear flap (ears flattened along head but without the lips thrust forward as with threat), and lip smack (lips repeatedly moving up and down, chattering of teeth). The occurrence of a particular behavior disturbance at least once during the food-reaching latency period was given a score of 1 . No further recording was made of the frequency of any additional incidents of that particular behavior disturbance during this latency period. Thus, the maximum composite emotionality score for any subject on any particular trial was 10 .

An evaluation of the real snake's movement was made upon each presentation, since it was not feasible to control for this variable. The rating system was based on a scale of increasing degree of movement from 0 to 3 , with 0 indicating no movement at all and 3 indicating that the snake was on the verge of coming out of the Plexiglas box. On no trial did the snake actually leave the box, although it was free to do so since the box was uncovered.

In all statistical analyses, the rejection level was set at $\mathrm{p}<.05$, unless otherwise indicated; all significant effects are reported.

\section{Results}

For each test object, the distribution of latency scores and the mean latency scores of each group (wild-vs. lab-reared) are presented in Table 1 and Figure 1. The latencies of the reaching responses were analyzed in a mixed-design analysis of variance. There were two between-groups variables (wild- vs. lab-reared, and order of stimulus presentation, i.e., toy vs. real snake first) and two within-groups variables (stimulus object presented, i.e., real snake, toy snake, model snake, yellow cord, black cord, and neutral object; and first or second presentation of a stimulus). For the neutral objects, an average of Trials 1 and 2 was used to represent the first neutral stimulus presentation and an average of Trials 12 and 13 was used to represent the second neutral stimulus presentation. The ANOVA revealed

Table 1

Frequency Distribution of the Average Latency Scores of the Reaching Response in Wild-Reared (W) and Laboratory-Reared (L) Animals

Average Latency Score Interval (in Seconds) for the Two Presentations of Each Stimulus

\begin{tabular}{|c|c|c|c|c|c|c|c|c|c|c|c|c|c|c|}
\hline \multirow[b]{2}{*}{ Object } & \multicolumn{2}{|c|}{$0-9.9$} & \multicolumn{2}{|c|}{$10-19.9$} & \multicolumn{2}{|c|}{$20-29.9$} & \multicolumn{2}{|c|}{$30-39.9$} & \multicolumn{2}{|c|}{$40-49.9$} & \multicolumn{2}{|c|}{$50-59.9$} & \multicolumn{2}{|c|}{60.0} \\
\hline & w & $\mathrm{L}$ & w & $\mathrm{L}$ & W & $\mathrm{L}$ & w & L & w & $\mathrm{L}$ & w & L & W & L \\
\hline Real & 2 & 8 & 1 & 0 & 0 & 0 & 1 & 0 & 0 & 0 & 0 & 0 & 6 & 2 \\
\hline Toy & 2 & 8 & 0 & 0 & 0 & 1 & 1 & 0 & 0 & 0 & 0 & 0 & 7 & 1 \\
\hline Model & 4 & 8 & 0 & 1 & 0 & 0 & 0 & 0 & 0 & 0 & 0 & 0 & 6 & 1 \\
\hline Black & 5 & 9 & 1 & 1 & 1 & 0 & 2 & 0 & 0 & 0 & 0 & 0 & 1 & 0 \\
\hline Yellow & 7 & 7 & 0 & 0 & 1 & 1 & 1 & 1 & 0 & 0 & 0 & 0 & 1 & 1 \\
\hline Neutral & 8 & 10 & 1 & 0 & 0 & 0 & 1 & 0 & 0 & 0 & 0 & 0 & 0 & 0 \\
\hline
\end{tabular}




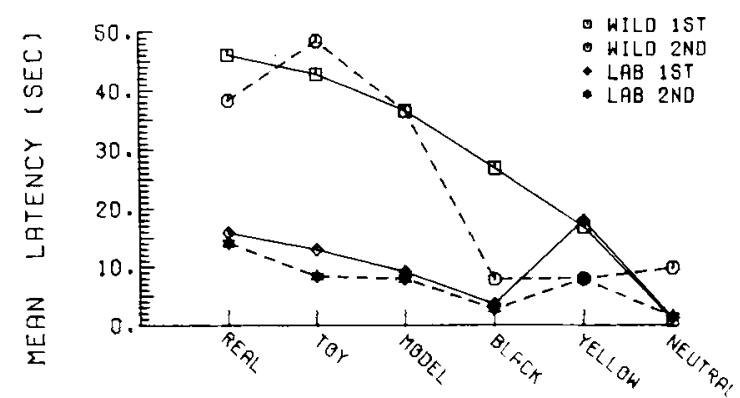

Figure 1. Mean latency to reach for the food in the wild-reared and lab-reared monkeys of experiment 1 on both the first and the second presentations of the six different objects (the two neutral objects are averaged together). The six objects are ordered as a function of the responses of the wild-reared monkeys 10 them.

a significant main effect of rearing condition $[F(1,16)$ $=8.20]$ and a significant Rearing Condition by Stimulus Object interaction $[F(5,80)=4.87]$. The interaction was the result of the wild-reared monkeys' having significantly longer latencies than the labreared subjects with the real snake $[F(1,16)=7.97]$, the toy snake $[F(1,16)=13.24]$, the model snake $[F(1,16)=5.72]$, and the black cord $[F(1,16)=$ 5.66], but not with the yellow cord or the neutral objects ( $F s<1.96)$. There was no significant effect of the order of presentation.

Among the within-groups variables, there was a significant effect of the stimulus object $[F(5,80)=$ 10.33] and a significant Stimulus Presentation (first vs. second) by Stimulus Object interaction $[F(5,80)$ $=3.201$. There was also a significant triple interaction between rearing condition, stimulus presentation, and stimulus object $[F(5,80)=2.65]$. As shown in Figure 1, the triple interaction appears to be primarily the result of the wild-reared monkeys' showing shorter latencies on the second presentation of the black cord than they did on the first.

Further analysis of the stimulus object main effect and the Rearing Condition by Stimulus Object interaction revealed no significant differences between stimulus objects for the lab-reared monkeys (Duncan multiple range tests, $a=.01$ ). The wildreared monkeys, by contrast, responded differentially to the various stimuli. Latencies were significantly longer to the toy snake than to the black cord, yellow cord, and neutral object; latencies were significantly longer to the real snake than to the yellow cord and the neutral object; and latencies were significantly longer to the model snake than to the neutral object (Duncan tests, $\alpha=.01$ ).

An analysis was also performed to investigate whether there were any significant changes in latency to the neutral objects across the test session. Of special interest was whether any aftereffects were apparent on neutral object trials that followed a snake trial (real, toy, or model). A 2 by 2 by 12 (order of stimulus presentation by rearing condition by repeated measures) ANOVA performed on the 12 neutral object trials revealed a significant effect only of the repeated measure $[F(11,176)=2.29]$. The 12 trial means were $.93,1.16,8.76,2.56,7.19,1.68$, $7.39,4.05,4.97,3.85,1.33$, and 1.01. Duncan tests revealed that the latencies were significantly longer on the first three neutral trials that followed the real, toy, and model snakes (Trials 3, 5, and 7 of the 12 neutral-object trials) than on some of the other trials; for example, Trial $3>1,2,4,6,11$, 12; Trial $5>1,2,12$; and Trial $7>1,2,11,12$. All these effects were, however, significant only with $a=.05$; because of the large number of comparisons being made, their reliability may be questionable. In sum, it seems that there may be some small aftereffects of the real, toy, and model snakes, but only after their first presentation.

Because of their skewed distributions (see Table 1), the latency scores were also subjected to nonparametric statistical analysis. The basic pattern of results obtained was identical to that of the ANOVA results, except that the differences in the reactions of the lab- and the wild-reared monkeys to the model snake and black hose were not significant (Us $\geqslant 28$, MannWhitney $U$ test). In addition, Wilcoxon tests performed on the latencies to the various stimuli (averaged across the two stimulus presentations) did reveal some small differences for the lab-reared monkeys which had not been significant with the Duncan tests. In fact, the response latency was significantly shorter to the neutral objects than to each of the other objects (all $T \leqslant 6$, Wilcoxon test).

Figure 2 shows the number of subjects in each group that showed any behavior disturbance when presented with the various objects (see Procedure section for a description of how the emotionality scores were obtained). Because the measure of behavior

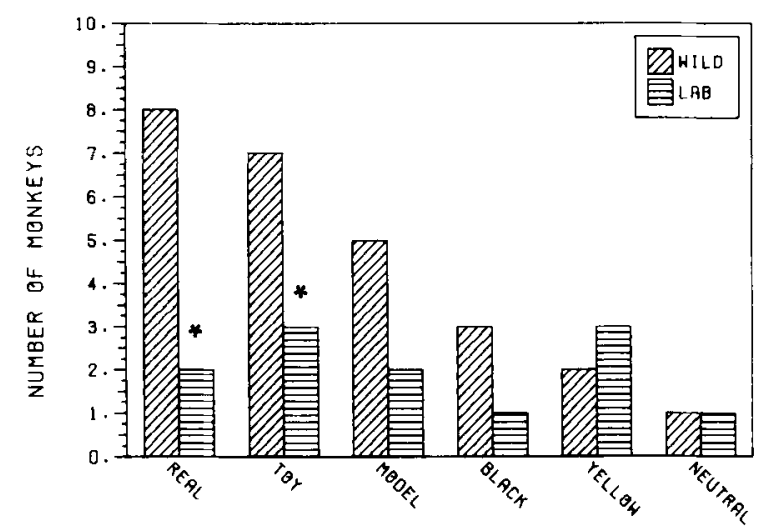

Figure 2. Number of wild-reared and lab-reared monkeys in Experiment 1 showing uny behavior disturbance to the six different objects. The * indicales that the wild vs. lab comparison is statistically significant. 
disturbance was not particularly sensitive (i.e., a score of 1 was given for a behavior that occurred any number of times during one latency period) and because many monkeys (especially lab-reared monkeys) showed no behavior disturbance at all, even with the snake objects, the emotionality scores were analyzed only with nonparametric statistics, with the scores obtained on the two presentations of an object summed together. In a comparison of composite emotionality scores, Mann-Whitney U tests revealed that the wildreared monkeys had significantly higher emotionality scores than the lab-reared monkeys for the real and toy snakes only (Us $=17.5$ and 22 , respectively). Friedman two-way ANOVAs conducted to analyze differences in emotionality scores across objects revealed that the wild-reared monkeys responded differentially to the various stimuli $\left(\chi_{r}^{2}=14.04\right)$, but that the lab-reared animals did not $\left(\chi_{\mathrm{r}}^{2}=1.94\right)$.

In an attempt to assess the effect of snake movement on response latency, Spearman rank correlations were computed separately for wild- and lab-reared subjects for both presentations of the real snake. No significant relationship was found between snake movement and response latency. This is probably not too surprising given the somewhat crude measure of, and limited range of possible scores for, snake movement. It should be noted, however, that on each of the four trials when the two lab-reared monkeys did show 60-sec latencies, the snake's movement was rated as 2 or 3 .

For the wild-reared monkeys, correlations were also computed between latency of the reaching response to a particular stimulus and the emotionality score for that stimulus. The average of each subject's two latency scores to the real, toy, and model snakes was correlated with the sum of the emotionality scores on the first and second presentations of each stimulus. Significant Spearman correlation coefficients were found between latency and emotionality for all three stimuli ( $\mathrm{r}_{S} \mathrm{~s}=.66, .88$, and .88 , for the real, toy, and model snakes, respectively). Parallel correlations could not be calculated for the lab-reared monkeys because there were so few trials on which emotionality scores were greater than 0 . It should be noted that these correlations may not be particularly meaningful because the range of emotionality scores on short-latency trials was limited by the opportunity to show disturbed behaviors; that is, a subject with a 10-sec latency had only $10 \mathrm{sec}$ in which to demonstrate behavior disturbance. Thus, the significant correlations for the wild-reared subjects might be confounded by the subjects' simply having a greater opportunity to display behavior disturbance on longlatency trials. (See Experiment 2 for further discussion of this issue.)

There were no obvious sex differences in the responses to the various stimuli, and there was nothing in the animals' life histories in the laboratory that appeared to affect their behavior during the experiment. One of the lab-reared monkeys that showed fear of the snake had participated in the Joslin et al. (1964) experiment approximately 15 years previously, but there was no way to ascertain whether or not she had shown fear then as well.

\section{Discussion}

The results of Experiment 1 confirm and extend those of Joslin et al. (1964). Eight of 10 wild-reared rhesus monkeys displayed a strong fear of either the live boa constrictor or the toy snake on at least one trial (7 did on both trials), whereas only 2 lab-reared monkeys showed similar reactions. Joslin et al. reported very similar proportions for their two groups. Thus, snake fear does not appear to be very common or pronounced in lab-reared monkeys, even when the snake is presented in an open box. Furthermore, the fear seen in the wild-reared monkeys does not appear to be simply a reaction to novelty, because the fear persisted across both stimulus presentations (except for the mild reaction seen to the black cord, which did diminish by the second presentation).

Our results differ slightly from those of Joslin et al. in that nonparametric Wilcoxon tests revealed that our lab-reared monkeys showed slightly longer latencies to all the snake-like objects than they did to the novel objects. Joslin et al. found a difference for their labreared monkeys only between the real snake and the neutral objects. These mild reactions may be the result of our having presented the objects in an open box rather than in clamps as Joslin et al. did. Alternatively, our having averaged across two neutral stimulus presentations may simply have increased the sensitivity of our test. Because Joslin et al. presented each stimulus only once and the neutral stimulus did not generally occur at the outset, their latencies to the neutral stimulus may have been inflated in some cases by aftereffects from the snake trials, which would minimize the opportunity to perceive the difference between their monkeys' reactions to the model and the neutral object.

The patterns of response latency and of disturbed behavior in the wild-reared monkeys in our experiment (Figures 1 and 2) comprise what looks like a generalization gradient, with the greatest fear being exhibited to the real and toy snakes and with less fear being exhibited to the model snake and the black and yellow cords. It is interesting to note that the pronounced fear reaction to the live boa constrictor exhibited by the monkeys occurred in spite of the fact that boa constrictors are not sympatric with rhesus monkeys. The apparent (although not statistically significant) stronger reaction to the toy snake than to the model snake is also noteworthy, since, to humans, 
the model snake is much more life-like than the toy snake, which is rather artificial looking. It seems likely that the relative sizes of the toy and model snakes is more important than the degree of realism, since the toy snake is approximately twice as thick as the model. ${ }^{1}$ This idea is consistent with pilot observations in our laboratory that indicated that wildreared monkeys did not show as consistent or pronounced a fear reaction to small live grass snakes as they did to the toy snake (or to the larger live boa constrictor). Other investigators have also noted the probable importance of the size of the snake as a partial determinant of the fear reaction (e.g., Lorenz, 1971).

\section{EXPERIMENT 2}

Over the past 10 years, there has been increasingly widespread use of flooding or response-prevention procedures in the treatment of human fears and phobias (e.g., Marks, 1972; Rachman, 1978). Analogous techniques have been shown also to be highly effective in hastening the extinction of learned avoidance responses in animals (e.g., Baum, 1970; Mineka, 1979; Riccio \& Silvestri, 1973). We are not aware, however, of any published attempts to reduce naturally occurring intense fears in animals by flooding-type procedures. In Experiment 2, we therefore examined the possibility of reducing snake fear in wild-reared monkeys with a flooding-like or "extinction" procedure. ${ }^{2}$

Following an initial assessment of fear reactions to all of the objects, as in Experiment 1, eight monkeys were subjected to seven flooding, or extinction, sessions with the real snake. In each of these sessions, they were required to meet a criterion of four consecutive trials with response latencies of $10 \mathrm{sec}$ or less. The last two of the seven sessions were follow-up tests at 10-day intervals to determine if there was any spontaneous recovery of the fear. Following the last session, a behavioral test with all the objects identical to those used in the initial assessment was also performed. The procedure used during the behavioral tests as well as during flooding improved on that used in Experiment 1 in two ways. First, all trials lasted 1 min (or sometimes more during flooding) even if a response of very short latency occurred. Thus, because the monkeys had an equal opportunity to engage in the behaviors on both kinds of trials, behavioral disturbance scores could be fairly compared across long- and short-latency trials. In addition, this procedure reduced the possibility that any changes in response latency across trials could be due to escape learning (a problem in Murray \& King's, 1973, experiment). Second, a modified frequency scoring procedure was used in which three separate assessments of behavioral disturbances were made every minute; that is, every $20 \mathrm{sec}$, the monkey received a score for the number of disturbance behaviors it had exhibited once in that 20 -sec period.

\section{Method}

\section{Subjects}

The subjects were two male and six female rhesus monkeys that had all been born in India and imported 13-25 years previously. Their ages ranged from approximately 17 to 30 years (mean $=24.6$ ). As described for Experiment 1, they had participated in a range of experiments over the years and had lived under a variety of conditions. Three had also been subjects in Experiment 1 and had thus had a small amount of fairly recent exposure to a snake and snake-like objects (the experiments were run approximately 1 year apart).

\section{Apparatus}

The apparatus was the same as that described for Experiment 1 , except that a different boa constrictor was used. It was approximately $3 \mathrm{ft}(91.5 \mathrm{~cm})$ long, and slightly larger in diameter $\left(1 \frac{1 / 4}{4}\right.$ in., or $3.2 \mathrm{~cm}$ ) than the one used in Experiment 1.

\section{Procedure}

Adaptation. Within 3 days before the first behavioral test, each subject was adapted to the apparatus and the procedure. The adaptation technique used was similar to that described for Experiment 1 , except that the first few trials of the session were carried out without the Plexiglas box. Each subject was given at least two adaptation sessions on which it had to meet a criterion of five consecutive trials with latencies of $5 \mathrm{sec}$ or less.

Behavioral Test 1. The procedure for the first behavioral test (BT-1) was like that described for Experiment 1, with the following exceptions. (1) The trial length was always $60 \mathrm{sec}$ rather than being determined by the latency of the subject's reaching response. On trials when the monkey's response latency was less than $60 \mathrm{sec}$, the stimulus object was left in front of the cage for the full $60 \mathrm{sec}$ and behavior disturbances were scored every $20 \mathrm{sec}$ with a 1-0 modified frequency system (Sackett, 1978); that is, any behavior that occurred once or more in a 20 -sec time block was given a score of 1. (2) A slight procedural change was introduced to help reduce the variation in snake movement. Rather than being pushed toward the subject at the start of every trial as the cage blind was raised, the Plexiglas box with the object in it was now placed directly in front of the cage before the start of every trial. This modification was made because the snake used here was somewhat more active than the one used in Experiment 1 and the movement involved in pushing the tray forward sometimes appeared to disturb the snake. (3) Food restriction was not used in this or in any other phase of the experiment because it was not found to be necessary to get consistent responding. (4) The list of disturbance behaviors was expanded to include the following behaviors: piloerection, stare (prolonged, fixed gaze into box from back half of cage), and abnormal posture (rare postures, e.g., tucking head between feet while standing). (5) Snake movement was scored on a 1-3 scale, with 1 indicating no movement, 2 indicating a small amount of movement, and 3 indicating considerable movement.

Flood 1. On the day following the behavioral test, each monkey received its first flooding session. All flooding sessions used only the real snake, and session length was determined by the performance of the subject. Each monkey had to reach a criterion of four consecutive trials with response latencies of $10 \mathrm{sec}$ or less. The trial length was also largely determined by the performance of the subject inasmuch as the snake was kept in front of the cage for at least $60 \mathrm{sec}$ and up to $480 \mathrm{sec}(8 \mathrm{~min})$ if necessary. If no response had been made in $480 \mathrm{sec}$, the trial was terminated by the experimenter. During each trial, behavior disturbances were scored every $20 \mathrm{sec}$ with the modified frequency system. Since every trial was at least $60 \mathrm{sec}$ long (even if a response had been made in less than $60 \mathrm{sec}$ ), at least three scores were available for 
every trial and a maximum of 24 scores were available on the 480 -sec trials. When a response occurred within a 20 -sec interval falling between 60 and $480 \mathrm{sec}$, a score was assigned for that interval on the basis of what had occurred up to that point in the interval.

Floods 2-5. Starting 10 days following the first flooding session, all monkeys were given four more flooding sessions on 4 consecutive days. The procedure was identical to that described for the first session, with each monkey being required to reach a criterion of four consecutive trials with response latencies of $10 \mathrm{sec}$ or less.

Floods 6 and 7. Ten days following the fifth flooding session, a follow-up sixth flooding session was given to test for spontaneous recovery. The session was identical to that described above for Sessions 1-5. Ten days following the sixth flooding session, one more follow-up flooding session (the seventh) was given, again to test for spontaneous recovery.

Behavioral Test 2. On the day following the seventh flooding session (35 days since BT-1), a second behavioral test (BT-2) was given with all seven stimulus objects. Trial lengths were always $60 \mathrm{sec}$, and the stimuli were delivered in the sequence described for BT-1. This test was used to assess whether any reduction in fear of the real snake that had occurred during flooding would generalize to the other snake-like objects.

\section{Results}

\section{Behavioral Test 1}

All eight monkeys demonstrated fear of the snake on the first behavioral test, as evidenced by their not responding within $60 \mathrm{sec}$ on at least three of the four presentations of the real and toy snakes. Three of the monkeys did respond on one toy or real snake trial but not on the other three trials; the other five monkeys failed to respond on all four trials. Five of eight also did not respond on either trial with the model snake. Friedman two-way ANOVAs, using the average response latency across the two presentations of each object, indicated that the monkeys responded differentially to the six objects $\left(\chi_{\mathrm{r}}^{2}=31.84\right)$, and subsequent Wilcoxon tests indicated that the monkeys responded more slowly to the real, toy, and model snakes than to the other three objects (all Ts $=0$, except for model vs. black, n.s.). They also responded more slowly to black than to yellow or neutral (Ts $\leqslant 1$ ), but yellow and neutral did not differ.

Analysis of the behavioral disturbance scores revealed considerable emotionality during the trials with the snakes (real, toy, and model). A Friedman twoway ANOVA, using the total disturbance behaviors summed across the two presentations of each object, revealed differential responding to the various objects $\left(\chi_{\mathrm{r}}^{2}=31.38\right)$. Subsequent Wilcoxon tests revealed that the emotionality scores to the real and toy snakes did not differ, but that they were higher than the scores to all other objects (all $\mathrm{Ts} \leqslant 2$ ). The monkeys also responded with greater emotionality to the model snake than to the black, yellow, and neutral objects (all Ts $\leqslant 2$ ). Responding to the black, yellow, and neutral objects did not differ.

As in Experiment 1, separate Spearman rank correlations were computed for the real, toy, and model snakes between latency and behavior disturbance. The total number of behavior disturbances for a given object (summed across both stimulus presentations and the three $20-\mathrm{sec}$ intervals on each trial) were correlated with the average latency to respond on the two presentations of that object. In each case, the $r_{S}$ was positive but not significant $\left(r_{S} s=.59, .36\right.$, and .55 for the real, toy, and model snake, respectively). Thus, the significant relationship seen in Experiment 1 between latency and behavioral disturbance was probably partly the result of the confound that existed there; that is, there was less opportunity to show behavior disturbances on short-latency trials than there was on long-latency trials.

\section{Flooding Sessions 1-7}

As shown in Figure 3, there was an overall tendency across sessions for a decline in latency to respond, in trials to criterion, and in total exposure time to criterion. The two follow-up sessions gave some evidence of spontaneous recovery on the latency measure.

A one-way repeated measures ANOVA, with the seven sessions as the repeated measure, on the average latency of each subject for each session revealed a highly significant main effect $[F(6,42)=22.5]$. (Trials on which the subject did not respond were counted as having a 480 -sec latency, and, because the number of trials to criterion differed across subjects and sessions, the number of observations contributing to each average latency score differed across subjects and sessions.) Duncan tests revealed that the average latency was greater for Session 1 than for Sessions 3-7 $(\alpha=.01)$. There was a small increase from Session 5 to Sessions 6 and $7(\alpha=.05)$, which did not differ significantly.

A one-way repeated measures ANOVA on number of trials to criterion for each session also revealed a significant effect $[F(6,42)=6.4]$. Duncan tests revealed that the monkeys took more trials to reach criterion on Session 1 than on Sessions 4-7 $(\alpha=.01)$. Sessions 6 and 7 did not differ significantly from Session 5 in trials to criterion.

The total exposure to criterion per session was also analyzed with a one-way repeated measures ANOVA $[F(6,42)=12.87]$. This measure reflects the total number of seconds of exposure per session that each subject received before meeting criterion. Because short-latency trials (e.g., less than $60 \mathrm{sec}$ ) always involved $60 \mathrm{sec}$ of exposure, this total exposure measure does not simply equal the sum of the latencies across the trials in a session. Duncan tests revealed that the monkeys took more exposure to criterion on Session 1 than on Sessions 3-7 $(\alpha=.01)$. Total exposure to criterion did not differ on Sessions 5, 6, and 7.

A one-way repeated measures ANOVA was also performed on the average of the total disturbance scores during the first minute for each session, and the analysis revealed no significant effect $[F(6,42)$ 

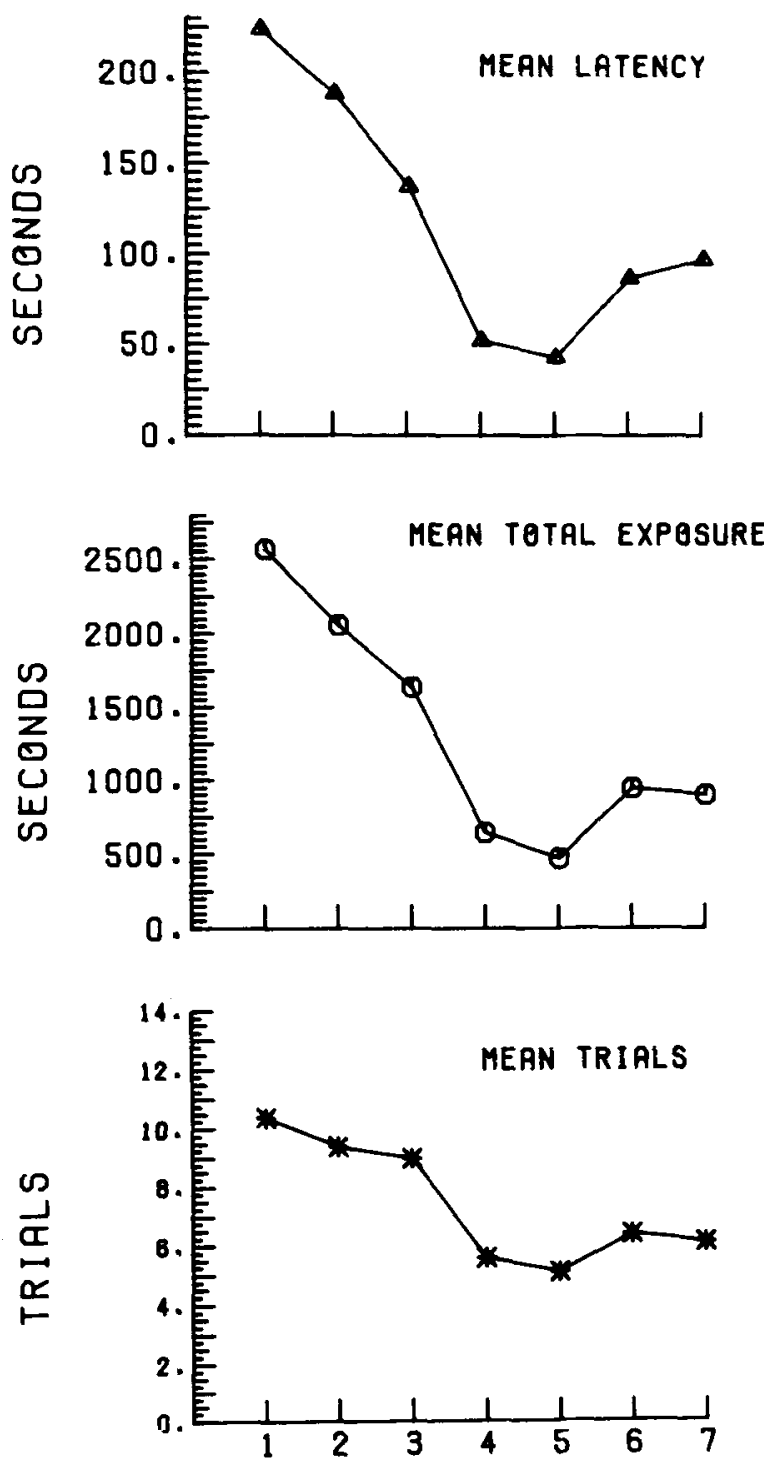

\section{SESSION NUMBER}

Figure 3. Mean latency, mean trials to criterion, and mean total exposure to criterion in Experiment 2 across the seven flooding sessions. There was a 10-day interval between Sessions 1 and 2, between Sessions 5 and 6, and between Sessions 6 and 7.

$=1.87$. The average disturbance score for a given subject on a given session was computed by summing the total number of disturbance behaviors that occurred in the first minute (summed across the three 20 -sec intervals) across all trials of that session and dividing by the number of trials for that subject in that session. When the same analysis was performed on the results from the first five sessions only, a significant effect was obtained $[F(4,28)=2.88]$. However, the significant effect did not appear to be due to a steady decline across sessions, but rather to the low mean behavioral disturbance on Session 3 alone. The means for the seven sessions were, respectively,
$4.7,4.5,2.9,4.4,4.0,3.6$, and 4.2. Thus, there was no systematic tendency that could be detected for the behavioral disturbance in the first minute to decline across sessions.

A number of within-sessions analyses were also performed. As indicated in Table 2, there was a bimodal distribution of latency scores across subjects and sessions; that is, the subjects tended to respond either in the first $60 \mathrm{sec}$ or not at all during the $480 \mathrm{sec}$ possible. The number of no-go trials $(480 \mathrm{sec}) \mathrm{de}-$ clined across sessions $[F(6,42)=9.42]$. The total number of no-gos were $33,25,17,4,3,10$, and 10 across Sessions 1-7, respectively. Subsequent Duncan tests revealed that there were more no-gos in Session 1 than in Sessions 3-7 $(\alpha=.01)$. Session 5 did not differ significantly from Session 6 or Session 7. Because of this go/no-go tendency characteristic of the data, two point-biserial correlations were computed to determine whether behavioral disturbance in the first minute was higher on no-go trials than on go trials. The first involved correlating the total behavioral disturbance score obtained in the first minute of each trial with trials having latencies $<60 \mathrm{sec}$ vs. trials having latencies $\geqslant 60 \mathrm{sec}$, collapsing across all subjects and trials of Session 1. A significant relationship was found $\left[r_{p b}=.39(n=83)\right]$, indicating that, in the first session, there tended to be more behavioral disturbance in the first minute on long-latency trials $(\geqslant 60 \mathrm{sec})$ than on short-latency trials $(<60 \mathrm{sec})$. The second correlation involved correlating trials with latencies $\leqslant 10 \mathrm{sec}$ vs. true no-go trials $(480 \mathrm{sec})$ with the total behavioral disturbance score obtained during the first minute of each trial, collapsing across all subjects and all sessions. (Trials with latencies between 11 and $479 \mathrm{sec}$ were omitted.) Again, a significant relationship was found $\left[r_{p b}=.39(n=375)\right]$, indicating that there was less behavioral disturbance in the first minute on trials with very short latencies than there was on trials in which the subject ultimately did not respond at all (480 sec latency).

Table 2

Frequency Distribution of the Average Response Latency During Flooding Sessions 1-7

\begin{tabular}{cc}
\hline $\begin{array}{c}\text { Response Latency } \\
\text { (in Seconds) }\end{array}$ & Frequency \\
\hline $480^{*}$ & 92 \\
$421-479$ & 2 \\
$361-420$ & 6 \\
$301-360$ & 2 \\
$241-300$ & 4 \\
$181-240$ & 4 \\
$121-180$ & 5 \\
$61-120$ & 10 \\
$0-60$ & $46 \dagger$ \\
$0-10$ & $19 \dagger$ \\
\hline
\end{tabular}

*No response.

tExcludes the last four criterion trials per session. 


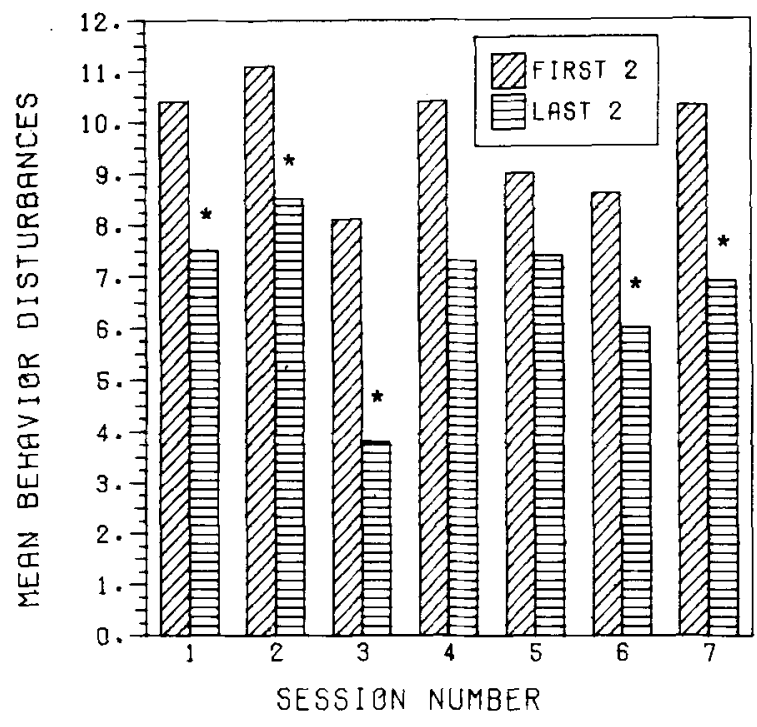

Figure 4. Mean behavioral disturbance scores for the tirst minute averaged across the first two and the last two trials of each of the seven flooding sessions of Experiment 2. I he * indicates that the first two vs. last two comparison is significant.

Although there was no systematic decline in behavioral disturbance across all sessions, there were changes within each session in the first-minute disturbance scores. Wilcoxon tests were performed to compare the behavioral disturbance on the first two trials of a session with that on the last two trials of the session. As shown in Figure 4, there was a significant drop within sessions for five of the seven sessions (all Ts $\leqslant 4$ for Sessions 1, 2, 3, 6, and 7). Thus, there was some evidence of habituation within most sessions of the behavioral disturbance which occurred during the first minute, although there was no systematic decline across sessions. An attempt was also made to determine whether there were changes in behavioral disturbance within the long no-go $(480 \mathrm{sec})$ trials. An examination for each subject of 2-6 no-go trials revealed no systematic changes in behavioral disturbance across the $8 \mathrm{~min}$ of each trial; that is, there was no systematic tendency for within-trial habituation of behavioral disturbance to occur.

\section{Behavioral Test 2}

The results from the second behavioral test (BT-2) are somewhat complex. At a simple level of analysis, there were three clinical "successes" and five clinical "failures"; that is, three monkeys responded rapidly to the real snake on both trials (successes) and five monkeys did not respond within $60 \mathrm{sec}$ to the real snake on either trial (failures). However, the results of the clinical "failures" were not uniformly negative, because four of the five responded to at least one object to which it had not on the first behavioral test, and the same four of five also showed overall less emotionality on all six snake trials (real, toy, and model) combined than they had on BT-1.
As a group, the monkeys still showed differential latencies of responding to the different objects, as they had on BT-1 $\left[\chi_{\mathrm{r}}^{2}=22.19\right.$, Friedman two-way ANOVA]. Subsequent Wilcoxon tests revealed that the monkeys had longer latencies to the real and toy snakes than to all other objects (all Ts $\leqslant 2$ ); latencies to the model snake and black cord were longer than to the yellow cord ( $\mathrm{Ts} \leqslant 2$ ). In addition, the monkeys responded with different amounts of emotional behavior to the different objects $\left(\chi_{\mathrm{r}}^{2}=20.92\right)$. Subsequent Wilcoxon tests revealed that the monkeys responded with greater emotionality to the real and toy snakes than they did to all other objects (Ts $\leqslant 1.5$ ). Thus, the general pattern of responding was similar to that seen in BT-1, although the behavioral disturbance response to the model snake no longer differed significantly from that to the other objects.

A number of statistical comparisons were made between the results of BT-1 and BT-2. The average latencies and behavioral disturbance scores to each object (averaged across the two stimulus presentations in each test) were compared with Wilcoxon tests. Only responding to the model snake (both latency of reaching and behavioral disturbance) changed significantly $(\mathrm{Ts} \leqslant 3)$. However, when a composite score reflecting the number of no-go responses $(60-\mathrm{sec}$ latencies) in the six snake trials (two each of real, toy, and model) was compared between BT-1 and BT-2, a significant difference was obtained $(T=0)$. Thus, seven of eight animals responded on the six snake-object trials with at least one fewer no-go response than they had on BT-1. In addition, composite emotionality scores to the same six objects declined significantly from BT-1 to BT-2 $(\mathrm{T}=3)$; the animals that showed this decline were the same as seven of eight that showed the decline in number of no-go trials. Thus, although the changes from BT-1 to BT-2 are not large when one considers individual objects, they are significant when one considers changes in number of no-go responses and in the overall amount of emotionality.

In spite of the small number of monkeys in the clinical success and failure categories, a concerted effort was made to determine whether any predictors of the success-failure dimension could be found. The two subgroups did not appear to differ in the severity of their fear on BT-1 (measured either by latencies or by behavioral disturbance). They also did not appear to differ substantially in the number of trials needed to reach criterion during flooding sessions, in average latency, in number of no-go trials, in average level of emotional disturbance, in habituation within sessions, or within trials, etc. The only thing that appeared to differentiate the two groups was the performance on follow-up Sessions 6 and 7. The clinical successes showed little sign of spontaneous recovery or return of fear, while four of the five 
clinical failures did show considerable spontaneous recovery. However, given that the performance on BT-2 is at least partially a test for spontaneous recovery as well as for generalization, it is not surprising to find that those monkeys showing return of fear at the first and second follow-up test should continue to do so in BT-2 (see Grey, Sartory, \& Rachman, 1979, for a discussion of the return of fear phenomenon). Finally, it may also be noted that all three successes were female, but, since three of the five failures were also female, no firm suggestions can be made about possible sex differences.

\section{Discussion}

The results of Experiment 2 demonstrate that the fear of snakes seen in wild-reared rhesus monkeys can be modified through prolonged exposure. In fact, all eight monkeys in our study successfully met a criterion of four consecutive short-latency responses within 6-18 trials (mean $=10.4$ ) in the first flooding session, and there was a significant drop in emotionality from the first two to the last two trials of that session as well. Thus, the results of Schiller (1952) with chimpanzees were confirmed with rhesus monkeys. However, our results go beyond those of Schiller by demonstrating that the changes that occur in one session do not mean that the fear has really been "abolished" or extinguished. Ten days later, a second flooding session proceeded almost exactly like the first, and it was not until the third or fourth session that changes in average latency, trials to criterion, and total exposure time began to decline from levels seen in the first session. Furthermore, performance on these latter sessions was by no means perfect; that is, reaching criterion on one day was only rarely followed by immediately reaching criterion in the next session. In fact, there were only 9 such instances, out of a total of 48 possible cases, in which a monkey met criterion immediately within 4 trials, and these 9 instances were accounted for by only five of the eight monkeys. In addition, there was little evidence of systematic changes in behavioral disturbance across the seven flooding sessions, even though, in most sessions, there was a significant within-session decline in emotionality. These results resemble those of Grey et al. (1979), who found considerable (but not complete) return of fear in their human phobics between the end of one flooding session and the beginning of the next.

Perhaps the most surprising aspects of the results of Experiment 2 are those of the second behavioral test. Even though all eight monkeys had reached a criterion of four consecutive trials with very short latency responses to the real snake in seven separate sessions, only three of the eight responded within
$60 \mathrm{sec}$ on the two real-snake trials of BT-2. A total of seven monkeys did show some change from BT-1 to BT-2, both in the number of no-go responses on the six snake trials and in total emotionality on those trials, but the changes hardly constituted an abolition of fear. Whatever the origins of this fear may have been, it seems to be very strongly ingrained in a good proportion of our wild-reared monkeys.

One final aspect of our results worthy of discussion is our observation that the average latency, the trials to criterion, and the total exposure time declined across flooding sessions, while average behavioral disturbance scores did not. This observation appears to be another case of the dissociation or desynchrony between different measures of fear that has been a topic of considerable discussion in recent years (e.g., Hodgson \& Rachman, 1974; Lang, 1968; Mineka, 1979; Rachman, 1978; Rachman \& Hodgson, 1974; Riccio \& Silvestri, 1973). These investigators have all noted that the three different response systems involved in fear (verbal/cognitive/subjective, behavioral avoidance, psychophysiological) do not always covary, and that treatments designed to reduce "fear" may, at least initially, affect one system and not the others. Flooding techniques have been noted to often produce a pattern of change similar to that observed here, that is, a change in the behavioral avoidance component without much concurrent change in the subjective or emotional component. In fact, Hodgson and Rachman (1974) noted: "It would appear that the first beneficial effect of flooding is an ability to control unwanted responses at the behavioral level. Autonomic and subjective signs of distress, associated with non-avoidance, are then gradually extinguished over a period of days, weeks, or months"' (p. 321). (See also Rachman, 1978.) In future work, it will be interesting to see whether more substantial changes can be produced in the emotional disturbance of our monkeys if they are subjected to even more prolonged flooding sessions, long after they have satisfied our behavioral approach criterion. It will also be interesting to compare flooding techniques with other more gradual techniques (e.g., counterconditioning) to determine whether the same degree of discordance or desynchrony occurs with the other techniques.

\section{REFERENCES}

BAUM, M. Extinction of avoidance responding through response prevention (flooding). Psychological Bulletin, 1970, 74, 276-284.

Grey, S., Sartory, G., \& Rachman, S. Synchronous and desynchronous changes during fear reduction. Behaviour Research and Therapy, 1979, 17, 137-148.

Harlow, H. F. The formation of learning sets. Psychological Review, 1949, 56, 51-65.

Haselnud, G. M. The effect of movement of stimulus objects upon avoidance reactions in chimpanzees. Journal of Comparative Psychology, 1938, 25, 507-528. 
Hевв, D. O. On the nature of fear. Psychological Review, 1946, 53, 259-276.

Hodgson, R., \& RACHMAN, S., II. Desynchrony in measures of fear. Behaviour Research and Therapy, 1974, 12, 319-326.

Joslin, J., Fletcher, H., \& EmLen, J. A comparison of the responses to snakes of lab-and wild-reared rhesus monkeys. Animal Behaviour, 1964, 12, 348-352.

LANG, P. J. Fear reduction and fear behavior: Problems in treating a construct. In J. M. Shlien (Ed.), Research in psychotherapy (Vol. 3), 1968.

LORENZ, R. Goeldi's monkey Callimico goeldii Thomas 1904 preying on snakes. Folia primatologica, 1971, 15, 133-142.

MARKs, I. Flooding (implosion) and allied treatments. In W. S. Agras (Ed.), Behavior modification: Principles and clinical applications. Boston: Little, Brown, 1972.

Masserman, J. H., \& Pechtel, C. Neuroses in monkeys: A preliminary report of experimental observations. Annals of the New York Academy of Sciences, 1953, 56, 253-265.

MinekA, $S$. The role of fear in theories of avoidance learning, flooding and extinction. Psychological Bulletin, 1979, 86, 985-1010.

Morris, R., \& Morris, D. Men and snakes. London: Hutchinson, 1965.

Murray, S. G., \& King, J. E. Snake avoidance in feral and laboratory reared squirrel monkeys. Behaviour, 1973, 47, 281289.

Rachman, S. Fear and courage. San Francisco: Freeman, 1978.

Rachman, S. The return of fear. Behaviour Research and Therapy, 1979, 17, 164-166.

Rachman, S., \& Hodgson, R. I. Synchrony and desynchrony in fear and avoidance. Behaviour Research and Therapy, 1974, 12, 311-318.

Riccio, D. C., \& Silvestri, R. Extinction of avoidance behavior and the problem of residual fear. Behaviour Research and Therapy, 1973, 11, 1-9.

SacketT, G. P. Measurement in observational research. In G. P. Sackett (Ed.), Observing behavior (Vol. 2). Baltimore: University Park Press, 1978.
Schille R, P. H. Innate constituents of complex responses in primates. Psychological Review, 1952, 59, 177-191.

Stephenson, G. R. Cultural acquisition of a specific learned response among rhesus monkeys. In D. Starck, R. Schneider, \& H. J. Kuhn (Eds.), Progress in Primatology. Stuttgart: Gustav Fischer Verlag, 1967.

Suomi, S. J. Social interactions of monkeys reared in a nuclear family environment versus monkeys reared with mothers and peers. Primates, 1974, 15, 311-320.

Wilson, G. T., \& Davison, G. C. Processes of fear reduction in systematic desensitization: Animal studies. Psychological Bulletin, 1971, 76, 1-14.

YERKES, R. M., \& YERKES, A. W. Nature and conditions of avoidance (fear) responses in chimpanzees. Journal of Comparative Psychology, 1936, 21, 53-66.

\section{NOTES}

1. It is interesting to note that the model used in this study is an exact replica in size of the live bullsnake used by Joslin et al. (1964). The mold for the model had been prepared some 15 years previously by making a plaster of paris impression of the live bullsnake used in that study. We reused the same mold to make our model.

2. Our procedure is not a pure flooding procedure in that food had to be used as an incentive for the monkey to respond and thus the procedure has certain elements in common with a counterconditioning procedure. However, the prolonged forced exposure to the snake makes the procedure overall much more like flooding than like counterconditioning (e.g., Wilson \& Davison, 1971). The term "extinction" is also used here with some hesitation, since it implies that the fear was conditioned in the first place; that is a point of which we cannot be certain.

(Received for publication September 4, 1979; revision accepted April 23, 1980.) 